\title{
Associations of specific types of sports and exercise with all-cause and cardiovascular-disease mortality: a cohort study of 80306 British adults
}

\author{
Pekka Oja, ${ }^{1}$ Paul Kelly, ${ }^{2}$ Zeljko Pedisic, ${ }^{3}$ Sylvia Titze, ${ }^{4}$ Adrian Bauman, ${ }^{5}$ \\ Charlie Foster, ${ }^{6}$ Mark Hamer, ${ }^{7}$ Melvyn Hillsdon, ${ }^{8}$ Emmanuel Stamatakis ${ }^{5}$
}

- Additional material is published online only. To view please visit the journal online (http://dx.doi.org/10.1136/ bjsports-2016-096822).

'UKK Institute, Tampere, Finland ${ }^{2}$ Sport, Physical Education and Health Sciences, University of Edinburgh, Edinburgh, UK ${ }^{3}$ Institute of Sport, Exercise and Active Living, Victoria University, Melbourne, Victoria, Australia

${ }^{4}$ Institute of Sport Sciences, University of Graz, Graz, Austria ${ }^{5}$ Sydney Medical School, University of Sydney, Sydney, New South Wales, Australia ${ }^{6}$ Nuffield Department of Population Health, University of Oxford, Oxford, UK

${ }^{7}$ School of Sport, Exercise and Health Sciences, Loughborough University, Loughborough, UK

${ }^{8}$ Sport and Health Sciences, University of Exeter, Exeter, UK

Correspondence to Dr Pekka Oja, UKK Institute, P.O. Box 30, Tampere 33501, Finland; pekka.oja@uta.fi

Accepted 11 October 2016 Published Online First 28 November 2016
ABSTRACT

Background/Aim Evidence for the long-term health effects of specific sport disciplines is scarce. Therefore, we examined the associations of six different types of sport/exercise with all-cause and cardiovascular disease (CVD) mortality risk in a large pooled Scottish and English population-based cohort.

Methods Cox proportional hazards regression was used to investigate the associations between each exposure and all-cause and CVD mortality with adjustment for potential confounders in 80306 individuals (54\% women; mean \pm SD age: $52 \pm 14$ years). Results Significant reductions in all-cause mortality were observed for participation in cycling $(\mathrm{HR}=0.85$, $95 \% \mathrm{Cl} 0.76$ to 0.95$)$, swimming ( $\mathrm{HR}=0.72,95 \% \mathrm{Cl}$ 0.65 to 0.80$)$, racquet sports $(\mathrm{HR}=0.53,95 \% \mathrm{Cl} 0.40$ to 0.69 ) and aerobics ( $\mathrm{HR}=0.73,95 \% \mathrm{Cl} 0.63$ to 0.85$)$. No significant associations were found for participation in football and running. A significant reduction in CVD mortality was observed for participation in swimming ( $H R=0.59,95 \% \mathrm{Cl} 0.46$ to 0.75$)$, racquet sports ( $H R=0.44,95 \% \mathrm{Cl} 0.24$ to 0.83 ) and aerobics ( $\mathrm{HR}=0.64,95 \% \mathrm{Cl} 0.45$ to 0.92 ), but there were no significant associations for cycling, running and football. Variable dose-response patterns between the exposure and the outcomes were found across the sport disciplines.

Conclusions These findings demonstrate that participation in specific sports may have significant benefits for public health. Future research should aim to further strengthen the sport-specific epidemiological evidence base and understanding of how to promote greater sports participation.

\section{BACKGROUND}

It is well established that physical activity (PA) has multiple cardiometabolic health benefits. ${ }^{1}$ This evidence comes largely from studies focusing on leisure-time PAs and active travel. ${ }^{2-5}$

Epidemiological cohort studies have suggested that sports participation is associated with reduced mortality among middle-aged and older adults. Samitz et $a l^{6}$ systematically reviewed 80 studies with 1338143 participants for associations between PA and risk of all-cause mortality. The domain 'vigorous exercise and sports' showed the largest reduction in risk of all-cause mortality $(\mathrm{RR}=0.78)$ followed by 'moderate and vigorous leisure-time activities' (RR 0.86), 'moderate activities of daily living' $(\mathrm{RR}=0.90)$, 'walking' $(R R=0.93)$ and 'PA for transportation' $(R R=0.92)$.
It has been suggested that vigorous-intensity PA, that is inherent to many types of sports and exercise, may have a higher impact on reducing allcause mortality risk than nonvigorous activities. Although sport is often cited as a contributor to public health, the nature and scope of this relationship remains unclear, particularly with regard to specific sport disciplines.

A recent systematic review of cross-sectional, cohort and intervention studies examined the health benefits of 26 specific sport disciplines. ${ }^{8}$ The most commonly studied sport disciplines were jogging/running, football, gymnastics, recreational cycling and swimming. According to established criteria for assessing the strength of evidence, ${ }^{9}$ there was moderate evidence for health benefits of jogging/running and recreational football and less than moderate evidence for all other sport disciplines. This review concluded that the existing evidence remains fragmentary and is compromised by weak study designs.

The aim of the present study was to examine: (1) the independent associations between participation in common types of sports and exercise and allcause and cardiovascular disease (CVD) mortality; and (2) the graded exposure-response characteristics of these associations in a pooled analysis of 10 general population cohorts of adults in England and Scotland.

\section{METHODS}

Sample

The Health Survey for England (HSE) and the Scottish Health Survey (SHeS) are household-based general population studies recruiting independent samples annually since 1991 (HSE) and periodically (SHeS) since 1995. Sampling is based on a multistage, stratified probability design aimed at a nationally representative sample of individuals living in households. ${ }^{10}{ }^{11}$ Interviewers visited the sampled households and administered the study questionnaire and measured height and weight. Each baseline data collection was approved by the relevant Research Ethics Committees in England and Scotland. All participants provided written consent to have their names flagged by the NHS Central Mortality Register. The present analysis included individuals aged 30-98 years from the HSE 1994, 1997，1998，1999，2003，2004, 2006 and 2008 and from the SHeS 1995, 1998 and 2003, with corresponding linkage to mortality data. For details of data acquisition and linkage see ref. 12 


\section{Physical activity assessment}

Non-occupational PA was assessed using an established questionnaire that inquired about the frequency and duration of participation in domestic PA (heavy manual housework, gardening and 'do-it-yourself' activities), walking and sports and exercise in the 4 weeks prior to the interview. ${ }^{13}$ Prompt cards were employed for sports and exercises that contained a number of groupings, including cycling (for any purpose), swimming, aerobics/keep fit/gymnastics/dance for fitness, running/jogging, football/rugby, badminton/tennis and squash. For each positive response participants were asked to specify frequency ('Can you tell me on how many separate days did you do [activity name] for at least 15 min a time during the past 4 weeks?'), duration ('How much time did you usually spend doing [activity name] on each day?') and perceived relative intensity ('Was the effort usually enough to make you out of breath or sweaty?'). The convergent validity of the questionnaire has been examined against accelerometry in a study of over 2000 adults. Spearman's correlation between questionnaire and accelerometer-based estimates of MVPA was 0.42 in men (95\% CI 0.36 to 0.48 ) and 0.38 in women $(95 \%$ CI 0.32 to 0.45$){ }^{13}$

\section{Mortality outcomes}

Surviving participants were censored on December 2009 (SHeS) or February 2011 (HSE). Primary causes of death were diagnosed according to the International Classification of Diseases (ICD) using the ninth (ICD-9) and tenth revisions (ICD-10). CVD codes recorded from the ICD were 390-459 and 101199 from the ninth and tenth revision, respectively (for details see ref. 12).

\section{Covariates}

Height and weight were measured using standard protocols that have been previously described; ${ }^{12}$ body mass index (BMI) was calculated as weight (in kilograms) divided by height (in metres) squared. Additional questions assessed education level (age finished education), weekly frequency of alcohol consumption, psychological distress/depression (12-item General Health Questionnaire score-GHQ-12), smoking status (current, ex, never), presence of limiting long-standing illness and existing doctor-diagnosed CVD (angina, stroke, coronary heart disease) and cancer.

\section{Data handling and statistical analyses}

The exposure measures were participation in cycling for any purpose (termed 'cycling'); swimming; aerobics/keep fit/gymnastics/dance for fitness (termed 'aerobics'); running/jogging (termed 'running'); football/rugby (termed 'football') and badminton/tennis/squash combined (termed 'racquet sports'). Other sports were considered but were not included in analysis, because of insufficient statistical power resulting from low participation rates. The associations between each exposure and mortality were examined in terms of:

A. overall participation (none/any);

B. relative perceived intensity (none/lower intensity/higher intensity) with the responses to the questions 'Was the effort usually enough to make you out of breath or sweaty?' determining lower versus higher intensity;

C. weekly duration (none/low/high) using the sex-specific medians of weekly times reported by participators for each exposure as cut-offs that denote low versus high duration (see online supplementary table S1 for cut-off values);

D. weekly intensity-weighted volume (metabolic equivalent (MET)-hours/week) calculated using the PA Compendium ${ }^{14}$ to assign a MET of activity and considering reported information on frequency, duration and relative intensity. Sex-specific medians of weekly volumes (MET-hours/week) were used as cut-offs that denote low versus high volume for each sport (see online supplementary table S1).

Baseline characteristics were summarised by sex. Cox proportional hazards regression was used to investigate the association between each exposure and all-cause and CVD mortality. Log-minus-log plots were used to examine proportional hazards assumptions. Selection of covariates was guided by previous literature and multivariate analyses were adjusted for age and sex (Model 1), and additionally adjusted for long-standing illness, alcohol drinking frequency, psychological distress (GHQ-12 score >3), BMI, smoking status, education level, doctordiagnosed CVD (all-cause mortality analyses only) and weekly PA volume (MET-hours/week excluding the volume of the sport that was the main exposure in the corresponding model). Participants who had doctor-diagnosed CVD at baseline (angina, stroke, IHD) were excluded from the analyses with CVD mortality as outcome. The linear and quadratic trend $p$ values were reported for each model. In sensitivity analyses, participants who experienced events occurring in the first 24 months of follow-up were excluded. Sensitivity analyses were also conducted to check the possible 'overadjustment' by BMI in Model 2. As no appreciable differences were found BMI was retained in the main analyses. No participation was always used as the reference category. Analyses were carried out with SPSS V.22 (SPSS, Chicago, Illinois, USA), with the level of statistical significance set at $\mathrm{p}<0.05$.

\section{RESULTS}

The baseline characteristics are shown in table 1. In total, our analysis included 80306 individuals with 43705 women (mean age $=52 \pm 15$ years) and 36601 men (mean age $=52 \pm 14$ years).

Overall, $44.3 \%$ of the participants $(43.1 \%$ of women and $45.6 \%$ of men) met national PA guidelines. The most common sport/exercise activity was swimming, followed by cycling, aerobics, running, racquet sports and football.

Over an average follow-up of $9.2 \pm 4.3$ years (corresponding to 736463 person-years), 8790 deaths from any cause occurred. Among the 75014 participants who did not report doctordiagnosed CVD at the baseline and were therefore entered in the CVD mortality analyses (9.2 \pm 4.5 years of follow-up/ 693757 person-years), there were 1909 CVD deaths.

\section{All-cause mortality}

Table 2 shows multivariate analyses of the association between exposure to specific sports and risk of all-cause mortality.

In the most adjusted model compared with no participation in each activity: cycling participation was associated with a significantly reduced risk of all-cause mortality of $15 \%(\mathrm{HR}=0.85$, $95 \%$ CI 0.76 to 0.95 ). Swimming participation was associated with a significantly reduced risk of all-cause mortality of $28 \%$ (HR $=0.72,95 \%$ CI 0.65 to 0.80 ). Running participation was not associated with a significant reduction in risk of all-cause mortality ( $\mathrm{HR}=0.87,95 \%$ CI 0.68 to 1.11 ). Likewise, football participation was not associated with a significantly reduced risk for all-cause mortality ( $\mathrm{HR}=0.82,95 \%$ CI 0.61 to 1.11$)$. Racquet sports participation was associated with a significant reduced risk of all-cause mortality of $47 \%(\mathrm{HR}=0.53,95 \% \mathrm{CI}$ 0.40 to 0.69 ). Aerobics participation was associated with a significant reduced risk for all-cause mortality of $27 \%(\mathrm{HR}=0.73$, $95 \%$ CI 0.63 to 0.85 ). 
Table 1 Baseline characteristics of the sample by sex

\begin{tabular}{|c|c|c|c|c|}
\hline & Women $(n=43705)$ & Men $(n=36601)$ & All $(n=80306)$ & $p$ value for $\operatorname{sex} \Delta *$ \\
\hline Age, mean $\pm S D$ (years) & $52.0 \pm 14.7$ & $51.9 \pm 14.3$ & $51.9 \pm 14.5$ & 0.285 \\
\hline Body mass index, mean $\pm \mathrm{SD}\left(\mathrm{kg} / \mathrm{m}^{2}\right)$ & $27.1 \pm 5.3$ & $27.3 \pm 4.0$ & $27.2 \pm 4.8$ & $<0.001$ \\
\hline Long-standing illnesst (\%) & 47.7 & 47.7 & 47.7 & 0.909 \\
\hline Smoking (\% current) $\ddagger$ & 24.3 & 25.5 & 24.9 & $<0.001$ \\
\hline Alcohol frequency $(\% \geq 5$ times/week $) \S$ & 14.7 & 25.3 & 19.5 & $<0.001$ \\
\hline Psychological distress (\% with GHQ score $\geq 4$ ) & 16.8 & 12.5 & 14.9 & $<0.001$ \\
\hline Age finished education (age 19+) & 16.3 & 20.2 & 18.1 & $<0.001$ \\
\hline Total physical activity volume, median (MET ${ }^{* *}$-hours/week) & 12.1 & 14.5 & 13.1 & $<0.001$ \\
\hline Meeting physical activity recommendations (\%) & 43.1 & 45.6 & 44.3 & $<0.001$ \\
\hline Cycling—any intensity (\% participated) & 7.3 & 13.0 & 9.9 & $<0.001$ \\
\hline Swimming—any intensity (\% participated) & 14.7 & 11.9 & 13.4 & $<0.001$ \\
\hline Running —any intensity (\% participated) & 3.1 & 7.3 & 5.0 & $<0.001$ \\
\hline Football—any intensity (\% participated) & 0.3 & 6.4 & 3.1 & $<0.001$ \\
\hline Racquet—any intensity (\% participated) & 2.7 & 4.8 & 3.6 & $<0.001$ \\
\hline Aerobics_-any intensity (\% participated) & 9.8 & 2.3 & 6.4 & $<0.001$ \\
\hline CVD prevalence at baseline $(\%)$ & 5.3 & 8.2 & 6.6 & $<0.001$ \\
\hline
\end{tabular}

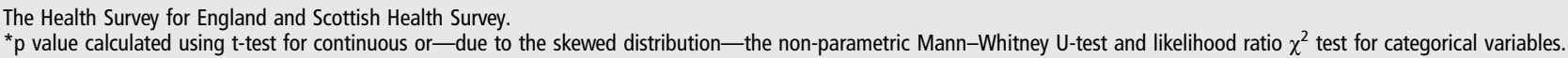

tDichotomous variable derived from responses to a series of questions (yes/no) on illness within eight listed body systems (eg, nervous system, digestive system, heart and circulatory system, etc), at least one illness required to have long-standing illness.

$\ddagger$ ‡ased on one question about smoking status with the options being: never, ex-regular smoker; ex-occasional smoker and current smoker.

$\S$ Derived from the question 'on how many days in the last 7 days did you have an alcoholic drink'.

१General Health Questionnaire comprises 12 questions related to psychological health (eg, concentration, feeling depressed, etc) with the categories: $0,1-3$ and $\geq 4$.

**MET, metabolic equivalent.

CVD, cardiovascular disease; GHQ, General Health Questionnaire.

\section{Cardiovascular disease mortality}

Table 3 shows multivariate analyses of the association between participation in specific sports and risk for CVD mortality.

In the most adjusted model compared with no participation in each activity: cycling participation was not associated with a reduced risk for CVD mortality ( $\mathrm{HR}=0.93$, 95\% CI 0.76 to 1.16). Swimming participation was associated with a significant reduced risk of CVD mortality of $41 \%$ ( $\mathrm{HR}=0.59,95 \% \mathrm{CI}$ 0.46 to 0.75 ). Running participation ( $\mathrm{HR}=0.81,95 \% \mathrm{CI} 0.47$ to 1.39 ) and football participation ( $\mathrm{HR}=0.90,95 \% \mathrm{CI} 0.49$ to 1.64) were not associated with a significantly reduced risk of CVD. Racquet sports participation was associated with a significant reduced risk of CVD mortality of $56 \%(\mathrm{HR}=0.44,95 \%$ CI 0.24 to 0.83 ). Aerobics participation was associated with a significant reduced risk of CVD mortality of $36 \%(\mathrm{HR}=0.64$, $95 \%$ CI 0.45 to 0.92 ).

\section{Dose-response analysis}

Online supplementary tables S2 and S3 show the results of the dose-response analyses for all-cause and CVD mortality, respectively. Mixed dose response relationships were obtained for different sport disciplines. Some showed a significant linear trend (indicating that reduction in mortality risk increased with higher intensity, duration and/or volume of sport participation), some showed a significant U-shaped relationship (indicating that lower intensity is more beneficial than high intensity or no participation), while some provided no indication of dose-response relationship. Fewer significant dose-response associations were found for CVD mortality compared with all-cause mortality.

\section{DISCUSSION}

In the present large population-based pooled cohort study, we examined the independent associations of the six most commonly practiced types of sport or exercise in Scotland and England with all-cause and CVD mortality. Swimming, aerobics and racquet sports were associated with significantly reduced risk of all-cause and CVD mortality. Cycling was associated with significantly reduced risk of all-cause mortality, but not CVD mortality. Our data did not show evidence of significant association with mortality risk for participation in running or football.

As noted, we conducted these analyses in response to a limited number of previous findings for specific sports/exercise. ${ }^{8}$ This limited the number of comparisons we could make.

Considering some notable results, at a specific activity level, our cycling and all-cause mortality result compares well with a 2014 systematic review and meta-analysis of eight studies that yielded a $10 \%$ risk reduction (95\% CI 6\% to 13\%) for cycling at a standardised dose of 11.25 MET-hours per week. ${ }^{5}$ We would suggest our result further confirms the likely magnitude of effect for cycling.

For swimming, a previous systematic review ${ }^{8}$ identified three cohort studies and one intervention study, but the evidence for all-cause mortality, CVD mortality and adiposity was inconclusive. In contrast, in the present study, swimming participation showed large and significant associations with all-cause and CVD mortality; $28 \%$ and $41 \%$ risk reduction, respectively.

The association between jogging/running and all-cause mortality among healthy adults has previously been addressed by four large-scale population-based cohort studies. ${ }^{15-18}$ The findings have been consistent in showing a significant risk reduction in all-cause and/or CVD mortality. The found significant reductions in all-cause mortality were $39 \%,{ }^{15} 30 \%,{ }^{16} 44 \%{ }^{17}$ and $27 \%$ and in CVD mortality $45 \% .{ }^{16}$ Our results showed a significant $43 \%(95 \%$ CI $27 \%$ to $55 \%)$ reduction in all-cause mortality risk among runners compared with non-runners according to the age/sex-adjusted model, but a non-significant $13 \%$ reduction in the fully adjusted model. A similar pattern was shown for CVD mortality. The fully adjusted associations in the present study are smaller than those reported in the previous studies, 
Table 2 Associations between sports participation and all-cause mortality in adults aged $\geq 30$ years $(n=80306$ )

\begin{tabular}{|c|c|c|c|c|}
\hline & Median age at death & Deaths/n & $\begin{array}{l}\text { Model 1* } \\
\text { HR }(95 \% \mathrm{CI})\end{array}$ & $\begin{array}{l}\text { Model } 2 \dagger \\
\text { HR }(95 \% \mathrm{Cl})\end{array}$ \\
\hline \multicolumn{5}{|c|}{ Cycling $\ddagger$} \\
\hline None & 77.0 & $8419 / 72373$ & 1.00 & 1.00 \\
\hline Any & 69.6 & $371 / 7933$ & $0.69(0.62$ to 0.77$)$ & $0.85(0.76$ to 0.95$)$ \\
\hline$p$ value & & & $<0.001$ & 0.003 \\
\hline \multicolumn{5}{|c|}{ Swimming } \\
\hline None & 77.0 & $8395 / 69525$ & 1.00 & 1.00 \\
\hline Any & 69.6 & $395 / 10781$ & $0.58(0.53$ to 0.65$)$ & 0.72 (0.65 to 0.80$)$ \\
\hline$p$ value & & & $<0.001$ & $<0.001$ \\
\hline \multicolumn{5}{|c|}{ Running§ } \\
\hline None & 77.0 & $8722 / 76294$ & 1.00 & 1.00 \\
\hline Any & 55.5 & $68 / 4012$ & 0.57 (0.45 to 0.73$)$ & $0.87(0.68$ to 1.11$)$ \\
\hline $\mathrm{p}$ value & & & $<0.001$ & 0.252 \\
\hline \multicolumn{5}{|c|}{ Footballף } \\
\hline None & 77.0 & $8747 / 77830$ & 1.00 & 1.00 \\
\hline Any & 54.0 & $43 / 2476$ & $0.63(0.47$ to 0.86$)$ & 0.82 (0.61 to 1.11$)$ \\
\hline $\mathrm{p}$ value & & & 0.003 & 0.175 \\
\hline \multicolumn{5}{|c|}{ Racquet sports** } \\
\hline None & 77.0 & 8736/77 391 & 1.00 & 1.00 \\
\hline Any & 66.0 & $54 / 2917$ & 0.38 (0.29 to 0.49$)$ & $0.53(0.40$ to 0.69$)$ \\
\hline$p$ value & & & $<0.001$ & $<0.001$ \\
\hline \multicolumn{5}{|c|}{ Aerobicst† } \\
\hline None & 77.0 & $8618 / 75165$ & 1.00 & 1.00 \\
\hline Any & 73.4 & $172 / 5141$ & $0.60(0.52$ to 0.70$)$ & 0.73 (0.63 to 0.85$)$ \\
\hline$p$ value & & & $<0.001$ & $<0.001$ \\
\hline \multicolumn{5}{|c|}{$\begin{array}{l}\text { *Model adjusted for age and sex. } \\
\text { †Model also adjusted for long-standing illness, alcohol drinking frequency, psychological distress (GHQ score), BMI, smoking status, education level, doctor-diagnosed cardiovascular } \\
\text { disease (IHD, angina, stroke) or cancer, and weekly volume of other physical activity (MET-hours, excluding the volume of the sport that was the main exposure in the corresponding } \\
\text { model). } \\
\text { †For any purpose. } \\
\text { §Running/jogging. } \\
\text { १Football/rugby. } \\
\text { **Badminton, tennis, squash. } \\
\text { †Aerobics/keep fit/gymnastics/dance for fitness. }\end{array}$} \\
\hline
\end{tabular}

and also statistically non-significant. This was a surprising finding, and we have considered a number of possible explanations. In our data, there were a relatively low number of mortality events in the exposure group which contributed to wide CIs and perhaps the non-significant HRs. Previous studies have also assessed running participation over longer recall periods ${ }^{16-18}$ than the current study. It might be that the recall period of 4 weeks used in the current study was not long enough to differentiate long-term and transient behaviour possibly resulting in misclassification of participants. It seems therefore that while not significant, our result adds to the body of evidence supporting beneficial effects of jogging/running on all-cause and CVD mortality rather than contradicting it.

Football showed a non-significant reduction in risk of allcause and CVD mortality. These non-significant associations were somewhat unexpected, as the evidence from controlled intervention studies in a systematic review indicates that participation in recreational football improves aerobic fitness and cardiovascular function at rest and reduces adiposity among previously inactive adults. ${ }^{8}$ Our finding may reflect the low prevalence of football in the study population $(0.3 \%$ among women, 6.4\% among men) and the consequent weaker statistical power in the analyses. We did additional analyses including only men and the HR (Model 2) remained non-significant for all-cause and CVD mortality (see online supplementary table S4).

Participation in racquet sports (including badminton, tennis and squash) showed significant risk reduction of $47 \%$ in allcause mortality and $59 \%$ reduction in CVD mortality. To the best of our knowledge little comparable data are available. A previous systematic review ${ }^{8}$ identified two studies on tennis and two on squash. One on tennis was a prospective cohort study, ${ }^{19}$ which showed decreased CVD risk among tennis players but no effect among racquetball players.

Strong and significant associations were found for all-cause and CVD mortality with aerobics participation (including aerobics, keep fit, gymnastics and dance for fitness). Additional analysis including only women indicated more marked reduction in CVD mortality compared with combined group analysis (50\% versus 36\%) (see online supplementary table S4) Aerobic dance has been shown to be associated with improved cardiorespiratory fitness ${ }^{20}$ and this in turn with reduced mortality risk. ${ }^{21}$ However, the direct link between participation in aerobic dance and mortality has not been previously investigated.

In order to place these observations in the context of overall PA, we have adjusted the analyses for meeting versus not meeting PA recommendations and for doing any sport versus doing no sport. The adjusted HRs for all-cause mortality were 
Table 3 Associations between sports participation and CVD mortality in adults aged $\geq 30$ years (excluding those with CVD at baseline, $\mathrm{n}=75$ 014)

\begin{tabular}{|c|c|c|c|c|}
\hline & Median age at death & Events/n & $\begin{array}{l}\text { Model 1* } \\
\text { HR (95\% Cl) }\end{array}$ & $\begin{array}{l}\text { Model } 2 \dagger \\
\text { HR }(95 \% \mathrm{Cl})\end{array}$ \\
\hline \multicolumn{5}{|l|}{ Cycling $\ddagger$} \\
\hline None & 76.0 & 1818/67 261 & 1.00 & 1.00 \\
\hline Any & 69.0 & $91 / 7753$ & 0.78 (0.63 to 0.97$)$ & 0.93 (0.76 to 1.16$)$ \\
\hline$p$ value & & & 0.023 & 0.533 \\
\hline \multicolumn{5}{|c|}{ Swimming } \\
\hline None & 76.1 & $1837 / 64486$ & 1.00 & 1.00 \\
\hline Any & 69.0 & $72 / 10528$ & $0.48(0.37$ to 0.60$)$ & 0.59 (0.46 to 0.75$)$ \\
\hline$p$ value & & & $<0.001$ & $<0.001$ \\
\hline \multicolumn{5}{|l|}{ Running§ } \\
\hline None & 76.0 & $1896 / 71026$ & 1.00 & 1.00 \\
\hline Any & 56.0 & $13 / 3988$ & 0.55 (0.32 to 0.93$)$ & 0.81 (0.47 to 1.39$)$ \\
\hline p Value & & & 0.026 & 0.451 \\
\hline \multicolumn{5}{|l|}{ Footballף } \\
\hline None & 76.0 & $1899 / 72558$ & 1.00 & 1.00 \\
\hline Any & 54.0 & $10 / 2456$ & 0.74 (0.41 to 1.35$)$ & 0.90 (0.49 to 1.64$)$ \\
\hline$p$ value & & & 0.325 & 0.736 \\
\hline \multicolumn{5}{|c|}{ Racquet sports** } \\
\hline None & 76.0 & 1900/72 131 & 1.00 & 1.00 \\
\hline Any & 66.0 & 9/2883 & $0.32(0.17$ to 0.60$)$ & 0.44 (0.24 to 0.83$)$ \\
\hline$p$ value & & & $<0.001$ & 0.011 \\
\hline \multicolumn{5}{|c|}{ Aerobictt } \\
\hline None & 76.0 & 1878/70 011 & 1.00 & 1.00 \\
\hline Any & 73.0 & $31 / 5003$ & $0.52(0.36$ to 0.74$)$ & 0.64 (0.45 to 0.92$)$ \\
\hline$p$ value & & & $<0.001$ & 0.015 \\
\hline
\end{tabular}

$0.73(0.69-0.77, \mathrm{p}<0.01)$ and $0.72(0.68-0.76, \mathrm{p}<0.001)$, respectively, and the corresponding HRs for CVD mortality $0.73(0.66-0.82, \mathrm{p}<0.001)$ and $0.72(0.64-0.80, \mathrm{p}<0.001)$. These results suggest that compared with meeting the generic PA guidelines (which also consider walking), doing any sport appears to be equally protective for all-cause and CVD mortality.

We tested for interaction by age (year 50 as cut-point), sex and PA level (meeting the PA recommendations versus not meeting them) of each sport participation by entering an interaction term in each fully adjusted model. With the exception of aerobics (sex interaction $\mathrm{p}=0.022)$ and cycling (PA level interaction $\mathrm{p}=0.036$ for all-cause mortality and $\mathrm{p}=0.012$ for CVD mortality), there were no interactions by age $(p \geq 0.155)$, sex ( $p \geq 0.101)$ or adherence to PA recommendations $(p \geq 0.211)$. Cycling appears to be more beneficial among inactive people for ACM and CVD (data available on request).

Mixed results were obtained for the dose-response relationship between participation in different types of sports and exercise and mortality. For running, we observed no significant dose-response gradient, which is in accordance with some previous findings. ${ }^{16}$ There was some indication of ' $U$ '-shaped doseresponse relationships between cycling, swimming and racquet sports participation and mortality, similar as in, for example, Schnohr $\mathrm{et} \mathrm{al}^{17}$ and Wang et al. ${ }^{18}$ There is an ongoing scientific debate about the shape of the dose-response relationship between PA and mortality, with strong arguments supporting both opposing views. Some contend that the relationship is ' $U$ ' shaped, ${ }^{22}{ }^{23}$ while others argue it is linear. ${ }^{24} 25$ Our results indicate variable patterns between the dose (intensity, weekly duration, weekly volume) of sport participation and the outcomes and highlight the need for further investigation.

It should be noted from tables 2 and 3 that the median age at death in groups with 'any' sport participation is considerably lower than in the 'none' groups. This could initially signal that participation leads to earlier death. However, we offer an alternative explanation of this phenomenon. At baseline, the median age for the 'any participation' groups is considerably lower than the 'none' groups. This means that after 10-year follow-up, the 'any' group is still considerably younger. This drives the phenomenon that the median age at deaths in the 'any' group is lower as it is derived from a younger sample.

The strengths of the present study include the large population representative sample with genders and a wide age range. To the best of our knowledge this is the largest existing data set reporting sport and exercise-specific associations with mortality 
risk. We used extensive national register-based mortality follow-up assessed independently. For the exposure assessment a validated questionnaire was used. The analyses controlled for a comprehensive set of covariates, although we cannot discount the possibility of residual confounding.

This study was not without limitations. First, the small number of events impaired the statistical power in some analyses. There were relatively few deaths due to all causes among runners and football players, which may explain the wide CIs in the final adjusted model. The number of CVD deaths was rather small among all sport/exercise participants and may reflect the weaker associations especially in the maximally adjusted model results. Nevertheless, the associations remained robust for swimming, racquet sports and aerobics. Second, seasonality remains an important issue in PA research, and particularly in our case investigating sports that may have defined on and off seasons. The surveys that we used employed sampling over a 12-month period to account for this issue, but some misclassification and resulting regression dilution may have reduced the strength of associations observed. Third, the relatively short recall period used for the assessment of the sport participation may have led to additional misclassification in terms of the long-term stability of the participation. Fourth, the repeat cross-sectional nature of the survey data available meant we could not assess or account for changes in participation within individuals. Finally, using mortality as an outcome may miss social and mental health benefits or reductions in morbidity conferred by sports participation.

The findings add to the existing body of knowledge suggesting that sport participation is likely to have important potential to promote public health. Future research should use welldesigned cohort studies to strengthen the evidence based on the associations between sport participation and mortality and morbidity, and also consider longitudinal changes in participation behaviour; conduct intervention studies to investigate if health benefits of these and other sport disciplines are truly causal in nature and find more effective ways of increasing population-level sports participation if the benefits are confirmed.

\section{CONCLUSIONS}

We found robust associations between participation in certain types of sport and exercise and mortality, indicating substantial reductions in all-cause and CVD mortality for swimming, racquet sports and aerobics and in all-cause mortality for cycling. The growing evidence should support the sport community to develop and promote health-enhancing sport programmes to reach more people and contribute to greater proportion of population meeting the PA guidelines for health.

\section{What are the findings?}

We found robust evidence that adults' participation in swimming, basketball and aerobics is associated with reduced all-cause and cardiovascular mortality and participation in cycling with that of all-cause mortality.

\section{How might it impact on clinical practice in the future?}

These new observations with other existing evidence should support the clinicians to consider sports participation as an effective form of health-enhancing physical activity.

Contributors PO and ZP conceived the idea for the study. PO and ES made a study plan. ES, PK, PO, ST and ZP contributed to the development of the data analysis design. ES processed and analysed the data. PO, PK, ST, ES and ZP drafted the manuscript. $A B, C F, M H a, M H i$ contributed to the writing of the manuscript. All authors contributed to drafting the rebuttal and revising the final draft of the manuscript.

\section{Competing interests None declared.}

Ethics approval Ethical approval was granted for all aspects of these studies by the following Ethics Committees prior to each survey year data collection: HSE 1994 was approved by the Medical Ethics Committee of the British Medical Association; HSE 1998/99 were approved by North Thames Multi Centre Research Ethics Committee; HSE 2003/2004 were approved by the London Multi-Centre Research Ethics Committee; SHS 1998 was approved by the Research Ethics Committees for All Health Boards for Scotland; SHS 2003 was approved by the Multi Research Ethics Committee for Scotland.

Provenance and peer review Not commissioned; externally peer reviewed.

\section{REFERENCES}

1 Lee IM, Shiroma EJ, Lobelo F, et al. Effect of physical inactivity on major non-communicable diseases worldwide: an analysis of burden of disease and life expectancy. Lancet 2012;380:219-29.

2 Hamer $M$, Chida Y. Walking and primary prevention: a meta-analysis of prospective cohort studies. Br J Sports Med 2008;42:238-43.

3 Woodcock J, Franco $\mathrm{OH}$, Orsini $\mathrm{N}$, et al. Non-vigorous physical activity and all-cause mortality: systematic review and meta-analysis of cohort studies. Int J Epidemiol 2011;40:121-38

4 Saunders LE, Green JM, Petticrew MP, et al. What are the health benefits of active travel? asystematic review of trials and cohort studies. PLoS One 2013;8:e69912.

5 Kelly P, Kahlmeier S, Götschi T, et al. Systematic review and meta-analysis of reduction in all-cause mortality from walking and cycling and shape of dose response relationship. Int J Behav Nutr Phys Act 2014;11:132.

6 Samitz G, Egger M, Zwahlen M. Domains of physical activity and all-cause mortality: systematic review and dose-response meta-analysis of cohort studies. Int J Epidemiol 2011;40:1382-400.

7 US Department of Health and Human Services. Physical Activity Guidelines Advisory Committee. Physical Activity Guidelines Advisory Committee Report. Washington, DC: $2008: 683$.

8 Oja P, Titze S, Kokko S, et al. Health benefits of different sport disciplines for adults: systematic review of observational and intervention studies with meta-analysis. Br J Sports Med 2015;49:434-40.

9 De Bourdeaudhuij I, Van Cauwenberghe E, Spittaels H, et al. School-based interventions promoting both physical activity and healthy eating in Europe: a systematic review within the HOPE project. Obes Rev 2011;12:205-16.

10 Mindell J, Biddulph JP, Hirani V, et al. Cohort profile: the health survey for England. Int J Epidemiol 2012:41:1585-93.

11 Gray L, Batty GD, Craig P, et al. Cohort profile: the Scottish health surveys cohort: linkage of study participants to routinely collected records for mortality, hospital discharge, cancer and offspring birth characteristics in three nationwide studies. Int J Epidemiol 2010;39:345-50.

12 Stamatakis E, Chau JY, Pedisic Z, et al. Are sitting occupations associated with increased all-cause, cancer, and cardiovascular disease mortality risk? a pooled analysis of seven British population cohorts. PLOS One 2013;8:26.

13 Scholes S, Coombs N, Pedisic Z, et al. Age- and sex-specific criterion validity of the health survey for England physical activity and sedentary behavior assessment questionnaire as compared with accelerometry. Am J Epidemiol 2014;179:1493-502.

14 Ainsworth BE, Haskell WL, Herrmann SD, et al. 2011 Compendium of physical activity: a second update of codes and MET values. Med Sci Sports Exerc 2011:43:1575-81.

15 Chakravarty EF, Huberts $\mathrm{HB}$, Lingala VB, et al. Reduced disability and mortality among aging runners. A 21-year longitudinal study. Arch Intern Med 2008;168:1638-46. 


\section{Original article}

16 Lee DC, Pate RR, Lavie CJ, et al. Leisure-time running reduces all-cause and cardiovascular mortality risk. J Am Coll Cardiol 2014;64:472-81.

17 Schnohr P, Marott JL, Lange P, et al. Longevity in male and female joggers. The Copenhagen city heart study. Am J Epidemiol 2013;177:683-9.

18 Wang N, Zhang X, Xiang Y-B, et al. Associations of Tai Chi, walking, and jogging with mortality in Chinese men. Am J Epidemiol 2013;178:791-6.

19 Chomistek AK, Cook NR, Flint AJ, et al. Vigorous-intensity leisure-time physical activity and risk of major chronic disease in men. Med Sci Sports Exerc 2012;44:1898-905.

20 Stofan JR, DiPietro L, Davis D, et al. Physical activity patterns associated with cardiorespiratory fitness and reduced mortality: the aerobics center longitudinal study. Am J Public Health 1998;88:1807-13.
21 Lee DC, Sui X, Ortega FB, et al. Comparisons of leisure-time physical activity and cardiorespiratory fitness as predictors of all-cause mortality in men and women. Br J Sports Med 2011;45:504-10.

22 O'Keefe JH, Schnohr P, Lavie CJ. The dose of running that best confers longevity. Heart 2013;99:588-90.

23 O'Keefe JH, Lavie CJ. Run for your life $\cdots$ at a comfortable speed and not too far. Heart 2013;99:516-19.

24 Arem $\mathrm{H}$, Moore SC, Patel A, et al. Leisure time physical activity and mortality: A detailed pooled analysis of the dose-response relationship. JAMA Intern Med 2015;175:959-67.

25 Gebel K, Ding D, Chey T, et al. Effect of moderate to vigorous physical activity on all-cause mortality in middle-aged and older Australians. JAMA Intern Med 2015;175:970-7. 